\title{
The airway microbiome of intubated premature infants: characteristics and changes that predict the development of bronchopulmonary dysplasia
}

\author{
Pablo Lohmann ${ }^{1}$, Ruth A. Luna ${ }^{2,3}$, Emily B. Hollister ${ }^{2,3}$, Sridevi Devaraj ${ }^{2,3}$, Toni-Ann Mistretta ${ }^{2,3}$, Stephen E. Welty ${ }^{1}$ and \\ James Versalovic ${ }^{2,3}$
}

BACKGROUND: Bronchopulmonary dysplasia (BPD) is associated with perinatal inflammatory triggers. Methods targeting bacterial rRNA may improve detection of microbial colonization in premature infants. We hypothesize that respiratory microbiota differs between preterm infants who develop BPD and those unaffected and correlates with inflammatory mediator concentrations.

METHODS: Twenty-five infants, born at $\leq 32$ wk of gestation and intubated in the first $24 \mathrm{~h}$, were enrolled. Tracheal aspirates were obtained at intubation and on days 3, 7, and 28. Bacterial DNA was extracted, and 165 rRNA genes were amplified and sequenced. Concentrations of interleukins (IL-1 $\beta, I L-6, \mid L-8$, IL-10, and IL-12), tumor necrosis factor-a, interferon- $\gamma$, lipopolysaccharide (LPS), and lipoteichoic acid (LTA) were measured. Chorioamnionitis was diagnosed by histology. BPD was defined as an oxygen requirement at 36 wk postmenstrual age. RESULTS: Acinetobacter was the predominant genus in the airways of all infants at birth. Ten infants developed BPD and showed reduced bacterial diversity at birth. No differences were detected in bacterial diversity, cytokines, LPS, and LTA from infants with and without exposure to chorioamnionitis.

CONCLUSION: The airways of premature infants are not sterile at birth. Reduced diversity of the microbiome may be an important factor in the development of BPD and is not associated with differences in inflammatory mediators.

B ronchopulmonary dysplasia (BPD) is a devastating form of chronic lung disease that develops in preterm infants. Despite advances in neonatal therapies and management strategies, the rates of BPD remain virtually unchanged, ranging from 50 to $80 \%$ in infants born at less than $28 \mathrm{wk}$ of gestation $(1-3)$. The primary factor leading to BPD is that the developmentally immature lung is susceptible to multiple injuries which interfere with pulmonary alveolar and vascular development and lung injury (4). Alterations in lung development due to insults cause long-term respiratory morbidity and are also associated with poor neurodevelopmental outcomes (5).
The mechanisms for development of BPD, specifically the lung injury, are multifactorial, not fully understood, and may be different from infant to infant, but lung inflammation may be a central mechanism to its development and progression (6-9). Several studies have shown associations between changes in diverse inflammatory markers and subsequent development of BPD (10-13). Among the various inflammatory triggers, bacterial colonization and/or infection have been implicated. The presence of certain organisms, whether the exposure is pre and/or postnatal, seems to play a potentially significant role (14-16). Multiple organisms have been implicated in the development of BPD, and attempts to intervene with antibiotics or anti-inflammatory agents, especially against Ureaplasma spp., have not changed the incidence of BPD (17-19). Presently, the gold standard for studies of infection or colonization is culture, and the sensitivity of microbial culture varies depending on the organism(s) being assessed. It is highly plausible that there are additional organisms that cannot be cultured effectively but are present in the respiratory tract and may contribute to the inflammatory process. This is especially important in premature neonates, who are predisposed to inflammatory tissue injury.

The airway microbiome is defined as the collective genomes of the microbial community that colonizes the respiratory tract and, currently, the composition of the normal neonatal respiratory microbiome has not been described. Cultureindependent methods for identification should avoid bias for organism detection due to media selection and offer higher sensitivity for both detection of individual organisms and complete community characterization than traditional culture methods $(20,21)$. The $16 \mathrm{~S}$ rRNA gene is a component of the prokaryotic ribosome and is highly conserved between different bacterial species. Next-generation sequencing, targeting the variable regions of the $16 \mathrm{~S}$ rRNA gene, allows for detection of less-abundant organisms in microbial communities and provides accurate identification to the genus level based on assignment of operational taxonomic units (OTU) $(22,23)$. 
Advances in high-throughput sequencing technology have allowed investigators to determine the taxonomic composition of intestinal microbiota (24), and ongoing research aims to obtain a better understanding of how the developing neonatal intestinal microbiome may affect pathologic processes, such as necrotizing enterocolitis. To date, there are very few studies that use culture-independent methods for detection of airway organisms in preterm infants, but there is evidence of temporal changes in the respiratory microbiome $(15,25-27)$. There are no published data regarding the respiratory microbiome in

Table 1. Demographic and clinical characteristics of enrolled infants

\begin{tabular}{lccc}
\hline & $\mathrm{BPD}(n=10)$ & No BPD $(n=12)$ & $P$ value \\
\hline GA (mean \pm SD) & $26.2 \pm 1.9$ & $28.9 \pm 1.4$ & 0.001 \\
Birth weight (g) (mean \pm SD) & $678 \pm 189$ & $1,284 \pm 360$ & 0.0001 \\
Male gender & $3(30 \%)$ & $7(58 \%)$ & NS \\
Preterm labor & $40 \%(4)$ & $50 \%(6)$ & NS \\
Antenatal steroids & $100 \%$ & $100 \%$ & NS \\
1 min Apgar (median) & $5(1-8)$ & $6(1-8)$ & NS \\
5 min Apgar (median) & $7(4-9)$ & $7(6-9)$ & NS \\
Surfactant doses (median) & $2(1-5)$ & $1(1-3)$ & NS \\
Cesarean delivery & $80 \%(8)$ & $92 \%(11)$ & NS \\
Presence of PIH & $50 \%(5)$ & $16 \%(2)$ & NS \\
PDA requiring treatment & $50 \%(5)$ & $25 \%(3)$ & NS \\
Chorioamnionitis & $40 \%(4)$ & $25 \%(3)$ & NS \\
Late sepsis & $30 \%(3)$ & $0 \%$ & 0.041 \\
\hline
\end{tabular}

BPD, bronchopulmonary dysplasia; GA, gestational age; NS, not significant; PDA, patent ductus arteriosus; $\mathrm{PIH}$, pregnancy-induced hypertension. premature infants and its potential relationship to bacterially mediated inflammation and development of BPD.

For this project, we hypothesized that respiratory microbiota would differ between ventilated preterm infants who develop BPD and those who do not develop BPD. A secondary hypothesis was that changes occur in the airways microbiome of ventilated premature infants in the early postnatal period and correlate with inflammatory marker concentrations and predict the development of BPD.

\section{RESULTS}

Twenty-five patients with gestational age (GA) ranging from 24 to 32 wk were enrolled. Seven patients were born to mothers with histological evidence of chorioamnionitis. Ten patients developed BPD. Over the course of the study, three patients died before day of life 5: two with Escherichia coli sepsis and one from extreme prematurity. One patient with BPD died from total bowel necrosis at 39 wks postmenstrual age. The data from this infant with BPD were analyzed with the BPD group as the bowel necrosis causing death was secondary to an incarcerated inguinal hernia, was not related to $\mathrm{BPD}$, and did not happen before $36 \mathrm{wk}$ postmenstrual age.

Demographic and clinical characteristics are shown in Table 1. There were no significant differences in the clinical and demographic characteristics of patients who did develop and did not develop BPD, except for GA, birth weight (BW), and culture-proven late sepsis. The three infants who died in the first month were not included in the demographics (mean GA: 25.4 wk, mean BW: $518 \pm 159 \mathrm{~g}$ ). All three mothers of those infants had severe pregnancy-induced hypertension.
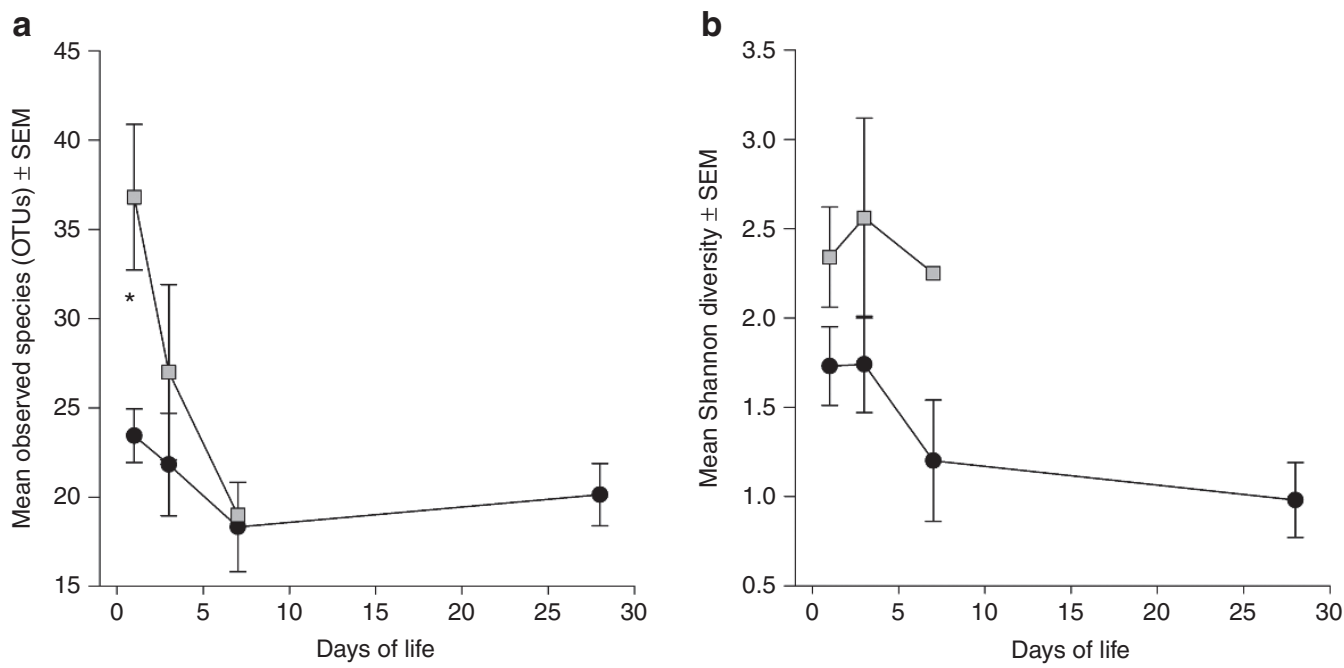

Figure 1. Infants who developed BPD exhibited decreased bacterial diversity. (a) Fewer bacterial species were observed in the BPD group (black circles), when compared with infants who did not develop BPD (gray squares). ${ }^{*} P=0.02$ at intubation. Mean observed species \pm SD for infants developing BPD: $23.4 \pm 4.5,21.8 \pm 7.03,18.3 \pm 6.12,20 \pm 4.6$; for infants without BPD: $36.8 \pm 12.91,27 \pm 8.5$, 19. (b) Lower Shannon diversity indexes were noted at time of intubation and persisted through day of life (DOL) 7 (when the last specimens were collected in the non-BPD infant). Mean Shannon index \pm SD for infants developing BPD: $1.73 \pm 0.65,1.74 \pm 0.66,1.2 \pm 0.82,0.98 \pm 0.56$; for infants without BPD: $2.34 \pm 0.87,2.56 \pm 0.97,2.25$. Number of infants who developed BPD $(n)=9,4,5$, and 7, at DOL 1 (intubation), DOL 3, DOL 7, and DOL 28, respectively, and number of infants who did not develop BPD $(n)=10,3$, and 1. BPD, bronchopulmonary dysplasia. 


\section{The Microbiome at Birth Differs Between Infants With and Without BPD}

All tracheal aspirate (TA) bacterial cultures obtained at time of intubation were negative; however, we were able to identify bacterial nucleic acid on all specimens obtained at birth. In two specimens, low levels of bacterial DNA were isolated; however, identifiable sequence could not be obtained, and those specimens were excluded from downstream analysis. After removing the $\mathrm{BW}$ effect, infants with and without $\mathrm{BPD}$ had significant differences in the diversity of their bacterial communities. More specifically, at time of intubation, neonates

Table 2. LPS, LTA, and cytokine levels at time of intubation (day of life 1) for infants exposed and not exposed to chorioamnionitis

\begin{tabular}{|c|c|c|c|}
\hline Biomarker & Chorioamnionitis & Not exposed & $P$ value \\
\hline LPS & $1.32 \pm 1.2$ & $2.26 \pm 2.1$ & 0.123 \\
\hline LTA & $320.1 \pm 149$ & $214.4 \pm 116$ & 0.189 \\
\hline IL-1 $\beta$ & $23 \pm 27$ & $6 \pm 8$ & 0.011 \\
\hline IL-6 & $2,895 \pm 1,120$ & $488 \pm 671$ & 0.890 \\
\hline IL-8 & $1,288 \pm 1,595$ & $556 \pm 717$ & 0.581 \\
\hline IL-10 & $756 \pm 292$ & $106 \pm 196$ & 0.001 \\
\hline IL-12 & $69 \pm 140$ & $37.88 \pm 67$ & 0.662 \\
\hline TNF- $\alpha$ & $57 \pm 31$ & $37 \pm 67$ & 0.010 \\
\hline IFN- $\gamma$ & $96 \pm 179$ & $17 \pm 23$ & 0.067 \\
\hline
\end{tabular}

who subsequently developed BPD had lower bacterial diversity, based on observed species count ( $23 \pm 4.5$ vs. $37 \pm 12$; $P=0.02)$ and Shannon diversity index values $(1.73 \pm 0.65$ vs. $2.34 \pm 0.87 ; P=0.094)$, than those who did not develop BPD (Figure 1a,b). Besides GA and BW, there were no other differing characteristics in the cohort.

\section{Chorioamnionitis and the Microbiome}

We compared the initial specimens of infants according to exposure to chorioamnionitis, and although the relative abundances of the dominant community members did not differ significantly between cohorts, we did note a trend toward decreased diversity in those infants who had been exposed. This is indicated by having 25 detectable species vs. 33 species in those unexposed infants and a lower Shannon diversity index of 2.04 vs. $2.23(P=0.19$ and $P=0.574)$. These trends were not statistically significant, and the observed differences in richness and diversity were largely a function of the nonchorioamnionitis communities harboring a greater variety of low-abundance organisms. When analyzing the log-transformed biomarker levels in infants exposed to chorioamnionitis, we noted significant differences in the levels of IL-1 $\beta$, IL-10, and tumor necrosis factor- $\alpha$. All the other biomarker levels did not differ significantly, and interestingly, at time of intubation, the levels of lipoteichoic acid (LTA) and lipopolysaccharide (LPS) were not statistically higher in infants exposed to chorioamnionitis (Table 2). Sequences corresponding to the intubation data point for two patients with chorioamnionitis were excluded from downstream analysis due to not meeting criteria for adequate analysis, as mentioned in the Methods section.
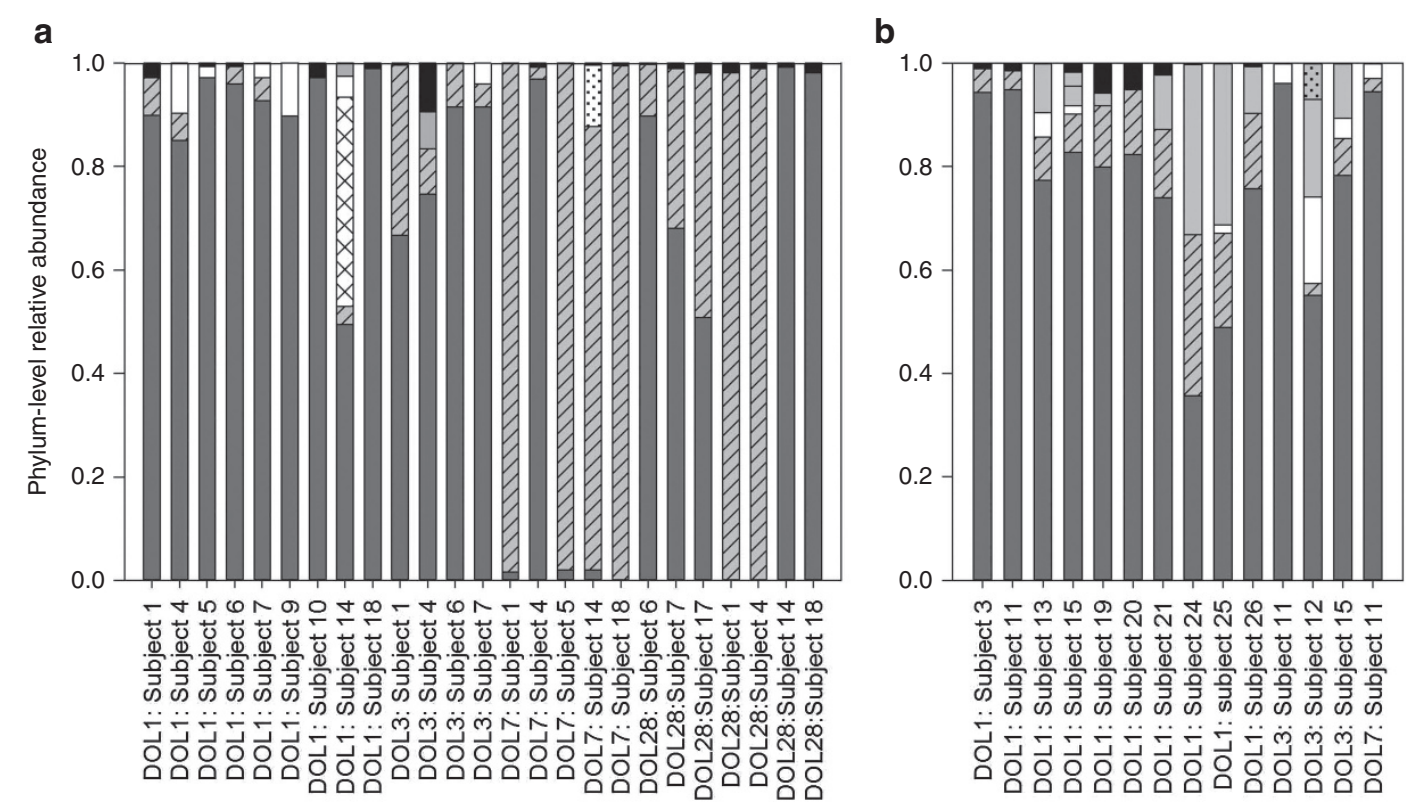

Figure 2. Phylum-level relative abundances of bacteria, over time, in the (a) airways of infants who developed BPD and (b) airways of infants who did not develop BPD. In the majority of BPD cases, the relative abundance of Proteobacteria declined over time, while the relative abundance of Firmicutes increased. In contrast, the bacterial communities in the non-BPD subjects in $\mathbf{b}$ remained relatively diverse and stable over time. Proteobacteria: dark gray; Firmicutes: light gray with diagonal stripes; Fusobacteria: white with cross-hatching; Tenericutes: white with stippling; Actinobacteria: white; Bacteroides: light gray; Cyanobacteria: light gray with stippling; and unclassified/other: black. BPD, bronchopulmonary dysplasia. 


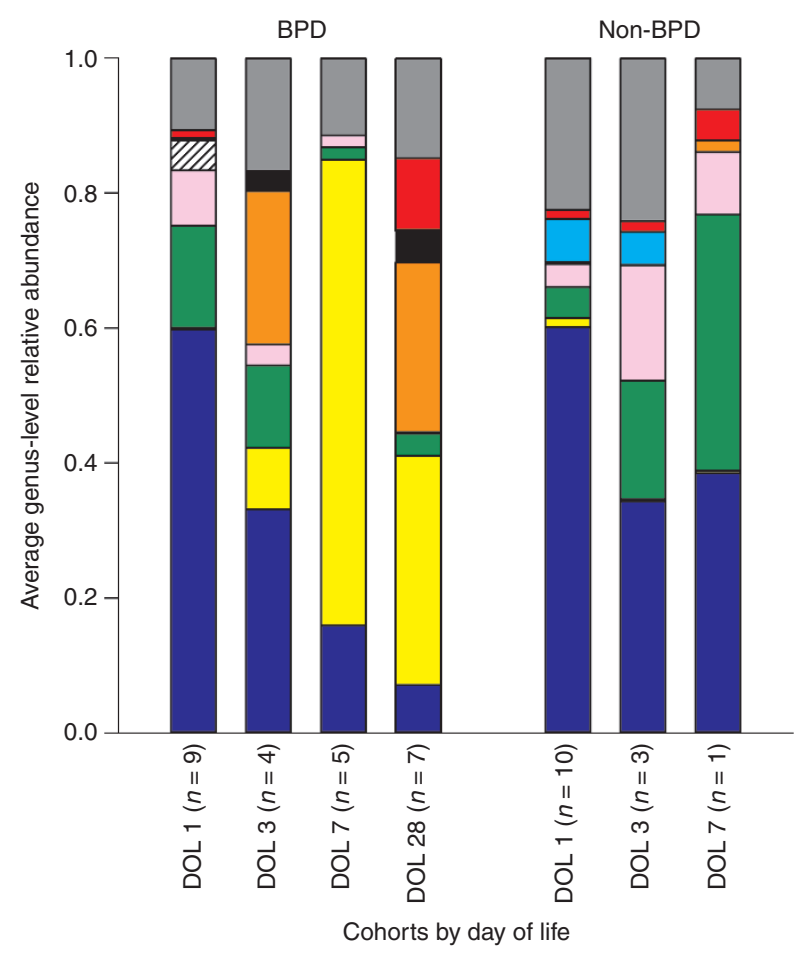

Figure 3. Average genus-level abundances of bacteria, over time, in the airways of infants who developed BPD and those who did not. Acinetobacter was the predominant genus in both groups at intubation. There is a shift in the relative abundance of species in those infants who developed BPD in contrast to the relatively diverse and stable community in the non-BPD group. The relative abundance of Acinetobacter sp. decreased longitudinally in the BPD group with increasing amounts of Staphylococcus and Klebsiella. The overall mean number of observed species and Shannon diversity index across time was significantly different in those with BPD than those without BPD $(P=0.042$ and $P=0.003)$. Acinetobacter: dark blue; Staphylococcus: yellow; Pseudomonas: green; Escherichia/Shigella: pink; Sneathia: white with diagonal stripes; Klebsiella: orange; Streptococcus: black; Bacteroides: light blue; Stenotrophomonas: red; and unclassified/other: gray. BPD, bronchopulmonary dysplasia.

\section{Evolution of the Microbiome Differs Between Infants With and Without BPD}

Phylum-level characterization of the microbial communities showed that Firmicutes increased and Proteobacteria decreased over time in those infants who developed BPD in contrast to the relatively diverse and stable community in the non-BPD group (Figure 2). No correlation was found between the levels of cytokines, LTA, and LPS and variations in the community composition. Twenty-three genera were identified in the BPD group with 36 genera identified in the nonBPD group. The overall mean number of observed species and Shannon diversity index across time was significantly different in those with BPD than those infants not affected $(P=0.042$ and $P=0.003$ ). Acinetobacter was the predominant genus in both groups, but the relative abundance of Acinetobacter sp. decreased longitudinally in the BPD group, with increasing amounts of Staphylococcus (OTUs matched to Staphylococcus epidermidis/Staphylococcus capitis, which cannot be distinguished from one another via 16S rRNA gene sequence) and Klebsiella (Figure 3). We found no statistical differences in the mean concentrations of cytokines, LTA, and LPS between the infants with and without BPD.

Two patients in the cohort developed late sepsis with positive blood cultures: one with Klebsiella pneumoniae and one with methicillin-resistant Staphylococcus aureus. Both patients developed BPD. Out of the cohort, 15 infants received empiric treatment with ampicillin and gentamicin for $48 \mathrm{~h}$; there were no significant differences in the initial specimen Shannon index of infants who received empiric antibiotic treatment and those who did not (data not shown).

\section{Case Analysis: Mortality}

A case-by-case analysis was performed in the infants who died, and no major differences were noted in the initial bacterial communities between these infants and the surviving infants. In one of the patients who died from E. coli sepsis, the community characterization from day of life 3 shows that $96 \%$ of the sequences recovered were from Escherichia/Shigella (Figure 4a). This patient had culture-proven bacteremia, and the necropsy showed disseminated infection.

One infant with BPD died from nonrespiratory causes. Longitudinally, the microbiome analysis in this patient showed decreasing richness, and the bacterial community was dominated by Staphylococcus spp., which composed 95\% of the recovered sequences by day of life 28 (Figure $4 \mathbf{b}$ ).

\section{DISCUSSION}

This study revealed that multiple bacterial taxa can be identified in the respiratory secretions of intubated premature infants, even at time of birth and prior to surfactant administration. This is interesting despite the fact that many of the infants in this study were delivered by cesarean section, with intact membranes and not in labor. This finding differs from the reported data by Mourani et al. (27), in which there was an insufficient quantity of bacterial DNA identified via sequencing prior to $72 \mathrm{~h}$ in most of the specimens. Our results are consistent with findings by DiGiulio et al. (28), who suggest associations between the presence of organisms or microbial DNA in amniotic fluid and clinical outcomes, such as preterm delivery. Furthermore, our results indicate that the amniotic sac is not a sterile environment, and it is highly likely that fetuses are exposed to a cohort of microorganisms prior to delivery regardless of the low-risk status such as intact membranes and absent labor. Interestingly, we can also measure LTA and LPS in initial TA secretions immediately after intubation. Detectable endotoxin levels in TAs might contribute to pulmonary inflammation, by triggering endotoxin-directed innate immunity, as noted by Nathe et al. (29). However, their study reports endotoxin levels in tracheal secretions after prolonged intubation and differs with our findings of detectable levels in 18 of the 25 (72\%) initial specimens. Unfortunately, there are no similar studies to which our data can be compared, so we cannot place our data in the context of previous reports. The measurements, however, are consistent with our findings indicating that along with bacterial DNA, we can isolate components of the wall of Gram-positive and Gram-negative 


\section{Articles | Lohmann et al.}
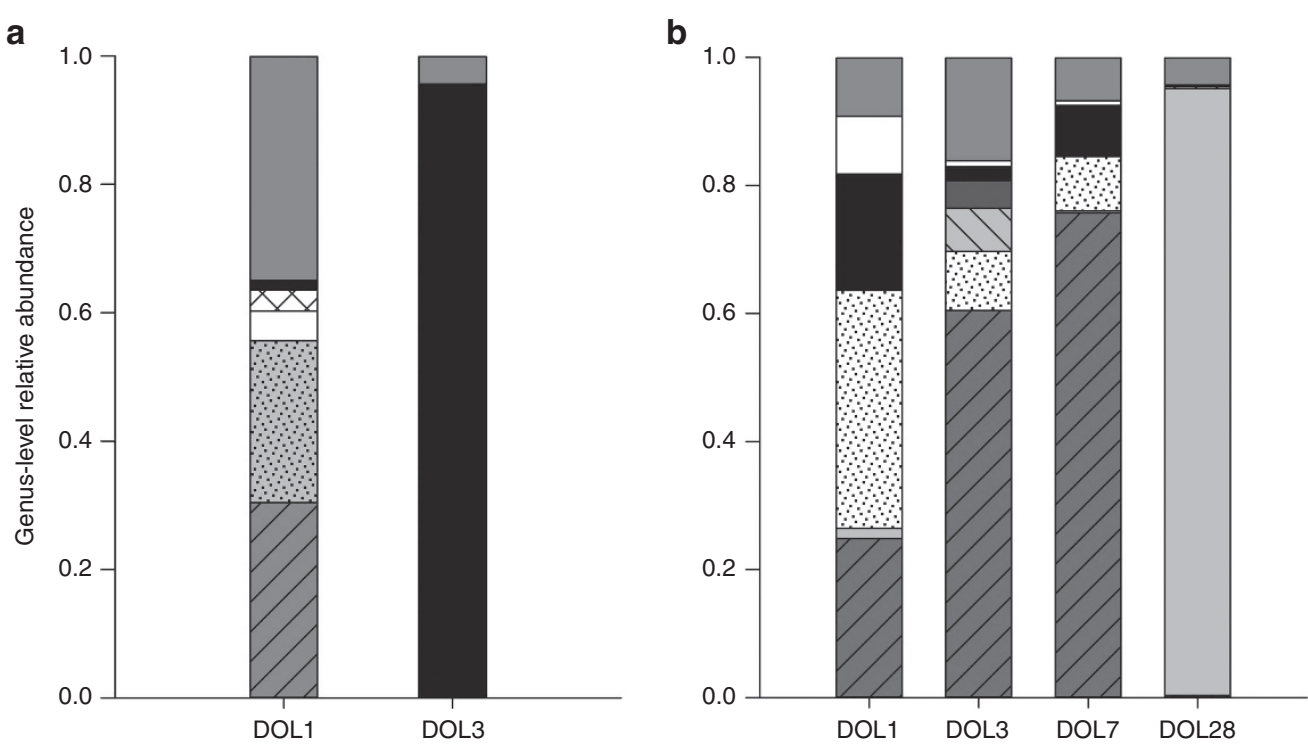

Figure 4. Genus-level relative abundances of bacteria in two cases of mortality. The infant profiled in panel (a) had culture-proven bacteremia and died of $E$. coli sepsis. The infant profiled in panel (b) died from nonrespiratory causes. However, over the first $28 \mathrm{~d}$ of life, this infant displayed progressive declines in community richness and diversity. Acinetobacter: medium gray with diagonal stripes; Bacteroides: light gray with stippling; Propionibacterium: white; Coprococcus: white with cross-hatching; Staphylococcus: light gray; Pseudomonas: white with stippling; Prevotella: light gray with diagonal stripes; Streptococcus: dark gray; Escherichia/Shigella: black; and unclassified/other: medium gray.

bacteria. It is possible that the evidence of bacterial presence is a by-product of contamination; however, we instilled sterile saline and aspirated secretions with a sterile catheter in attempts to minimize or eliminate contamination.

Perhaps the most impressive finding in the present study was that based on observed species counts and Shannon diversity index, there were notable differences between the respiratory microbiome of infants who subsequently developed BPD and those without chronic lung disease. The Shannon diversity index is a function of the number of species detected in a sample (i.e., richness) and the distribution of their relative abundances (i.e., evenness). High values of the Shannon index are typically the result of a species-rich and relatively evenlydistributed community, whereas low values of the Shannon index often reflect communities that are less species rich and may be dominated by a few predominant taxa. Figure 1 shows a difference in diversity initially, and the difference becomes less pronounced at $7 \mathrm{~d}$ of life. The Shannon index indicates lower diversity in the microbiome of infants that develop BPD, independent of the exposure to chorioamnionitis. This difference is observed despite the dropout of infants in the non-BPD group because of extubation and decreases with intubation time. While we cannot say for certain, as we did not collect specimens from patients once they were extubated, it is likely that the patients most easily extubated have the most favorable microbiome. If it were possible to include data from them, we expect that the differences would be more profound than limiting the analysis to intubated infants. This difference in diversity represents an apparent quantitative difference in infants that may be predictive of the development of BPD and may in fact be linked mechanistically. However, the present data set cannot address this possibility specifically. Qualitatively, Acinetobacter was present in all of the initial specimens, and the predominance of Acinetobacter sp. has not been reported previously in studies of the respiratory microbiome. We speculate that Acinetobacter presence may condition the way in which the respiratory tract is subsequently colonized. Neonatal gastrointestinal microbiome studies have shown immune modulation triggered by interactions between bacterial communities and the intestinal mucosa (30-32). It is possible that the respiratory tract is affected in a similar way by exposure to colonizing bacteria, which promote further seeding by subsequent species, a phenomenon known as microbiome succession.

In our data, it is important to note that the BW and GA of the infants who developed BPD were significantly lower than the BW and GA of the unaffected peers, and this raises the question of variable bacterial communities with advancing GA. Because of the size of the study and individual variations, we cannot conclude that the differences in the microbiota in infants who developed BPD and those who did not are cause and effect rather than a difference in the microbiome secondary to GA. Hence, this finding is most intriguing and requires future study.

We noticed a trend toward decreased diversity in the initial specimens of infants exposed to chorioamnionitis when compared with the unexposed infants ( 25 vs. 33 species). However, this difference was mostly due to a subset of relatively low-abundance organisms in the cohort that was not exposed to chorioamnionitis. The goal of this pilot study was to attempt to detect any microbial communities in these early life specimens and thus not powered toward detecting changes in cytokine levels. However, the lack of correlation between LPS, LTA, and presence of bacteria suggests that the differences noted in the levels of IL- $1 \beta$, IL-10, and tumor necrosis factor- $\alpha$ might be related to the inflammatory process itself independent of signaling by 
LPS or LTA. Toll-like receptors 2 and 4 constitute an important defense mechanism by mediating bacteria-induced inflammation in response to LTA and LPS, respectively (33). The lack of statistical differences in the levels of LTA and LPS between infants exposed and not exposed to chorioamnionitis suggests that the increase in cytokine levels is independent of the Tolllike receptor 2 and 4 pathways. Without major differences in the microbiome pattern, we speculate that chorioamnionitis leads to an increase in IL-1 $\beta$, IL-10, and tumor necrosis factor- $\alpha$ independent of signaling through LPS and LTA, although we cannot exclude a type 2 error because of small numbers of samples.

When analyzing specimen sets in a longitudinal fashion, there was a marked decrease in the diversity and number of species in the respiratory microbiome of infants who developed BPD. This does not appear to be a function of length of intubation, since for the same time points, there is no such decrease in the unaffected infants. We noted a significant increase in the relative abundance of Staphylococcus (OTUs matched to $S$. epidermidis/S. capitis) and Klebsiella, which is associated with a decrease in Acinetobacter. In contrast, those infants who did not develop BPD continued to have a relatively stable and resilient microbiome, without shifts in the dominant species. The diversity and species number also remained higher in the less sick infants, findings that suggest that a diverse microbiota is associated with healthier states, as noted in other studies $(34,35)$. Although it is hard to determine a causal relationship between the respiratory microbiome evolving toward a few heavily abundant species, it seems that such changes early in the course may be associated with BPD development.

\section{Conclusions}

Characterization of the respiratory microbiome at delivery suggests that neonatal airways are not sterile at intubation and that infants are colonized by a wide array of organisms, even without classic risk factors for colonization. The microbial patterns did not differ significantly despite exposure to chorioamnionitis. We noted that the evolution of the respiratory microbiome, with decreasing diversity of the bacterial community, is associated with BPD. We used biomarker levels as a proxy for an inflammatory and diseased state at the time of delivery, which was not confirmed, as LTA, LPS, and cytokine levels were not predictive of microbial community differences or shifts in composition as a function of time. The pathogenesis of BPD is complex, and these findings suggest that the initial exposures to bacterial products may not be central to the lung injury but rather affect the immune response and microbiome succession. High-throughput sequencing technology has allowed us to identify a possible clinically relevant microbiome in premature infants, but further studies are needed to improve our understanding of the bacteria-host interactions to enable interventions in the sequence of lung injury.

\section{METHODS}

\section{Study Population}

The study was performed as a prospective observational cohort design, and we obtained approval from Institutional Review Board of the Baylor College of Medicine. We included infants born at $\leq 32 \mathrm{wk}$ of GA, who underwent endotracheal intubation and mechanical ventilation in the first $24 \mathrm{~h}$ of life. Informed consent was obtained from either or both parents on notification of risk of premature delivery and/or admission to the neonatal intensive care unit at the Texas Children's Hospital (Houston, TX).

Infants with major congenital anomalies, congenital sepsis, or evidence of pulmonary hypoplasia were excluded. Outborn infants intubated prior to transfer to the neonatal intensive care unit at the Texas Children's Hospital were also excluded.

\section{Specimen Collection}

TA specimens were obtained in the delivery room if the infant met clinical indications for intubation (apnea or respiratory distress syndrome) and when tracheal suctioning was indicated clinically, per unit protocol. Longitudinal specimens were collected at the time of intubation (within $24 \mathrm{~h}$ of delivery) and days of life 3,7 , and 28 , if the infants were still intubated at those time points. The protocol for TA collection consisted of instillation of $0.5 \mathrm{ml}$ of sterile isotonic saline into the infant's endotracheal tube, manual bagging through the endotracheal tube for three breaths, and suctioning of the fluid into a sterile mucus trap. This procedure was repeated for a total of three times. This protocol has been utilized in previous studies of TA specimens in susceptible neonates $(10,11)$. After the specimen was collected, separate aliquots were made for bacterial DNA extraction, analysis of inflammatory mediator concentrations, and presence of bacterial cell wall products. An aliquot from the first specimen obtained from each patient was also sent for conventional microbiology culture.

\section{Specimen Processing and Analysis}

TA culture was performed per our microbiology laboratory standard for patients without cystic fibrosis. Three media plates, blood agar (sheep's blood), chocolate agar, and MacConkey agar, were inoculated with a fresh aliquot of the initial TA specimen and kept for $96 \mathrm{~h}$. A comprehensive report detailing all bacteria present was to be given for positive growth, whether normal oral/respiratory flora or an actual pathogen.

Bacterial DNA was extracted from each TA specimen using the Mo Bio PowerSoil kit (MO BIO Laboratories, Carlsbad, CA) with modifications used in the Human Microbiome Project (36). Sequence libraries were constructed by amplification of the V3-V5 regions of the bacterial 16S rRNA gene (V3V5-357F: 5' CCTACGGGAGGCAGCAG 3', V3V5-926R: 5' CCGTCAATTCM TTTRAGT $3^{\prime}$ ). Primers also contained the 454-based adapter (CCATCTCATCCCTGCGTGTCTCCGAC) as well as the key sequence (TCAG) followed by a unique identifier per sample, as suggested by the manufacturer. Negative controls (water) and positive controls (previously sequenced samples) were utilized throughout the extraction and amplification process and performed as expected. A no-template control was also included in the amplification and sequencing steps to allow for the evaluation of potential contamination. Utilizing Roche 454 sequencing technology, an average of 3,000 reads per region was obtained with an average read length over 500 bases. The no-template control, however, yielded no sequence reads. Resulting sequences were quality filtered and analyzed using QIIME (version 1.3.0; http://qiime.org/) (37), as implemented on the Genboree Microbiome Workbench (38).

Sequences shorter than 200 base pairs in length, possessing average quality scores $<20$, containing ambiguous base calls, or including mismatches to their barcode or primer sequences were filtered and excluded from downstream analysis. Sequences were clustered into OTUs using Cd-HIT at a 97\% similarity threshold (39). Alpha diversity metrics, including OTU richness and the Shannon diversity index, were calculated for each sample using QIIME. OTU richness refers to the number of unique OTUs identified within a sample, while the Shannon diversity index places a numeric value on its complexity (40). In order to account for potential biases associated with differences in sampling effort (i.e., sequencing depth), sequence libraries were randomly subsampled to an equal number of sequence reads $(1,012$ reads per sample) prior to analysis.

The primary analysis was the characterization of the respiratory microbiome via conventional culture and next-generation sequencing. Secondary analysis focused on the variability of the microbial 
community in a longitudinal fashion, as well as associations with cytokines and bacterial product concentrations. Bacterial composition (assignment and identification of OTUs) and bacterial diversity (measured with the Shannon diversity index) were determined for all specimens.

Data collection was performed via electronic medical record review at the time of enrollment, specimen collection, and during remainder of hospitalization.

Chorioamnionitis was determined by histopathology, BPD, defined as an oxygen requirement at $36 \mathrm{wk}$ postmenstrual age, via oxygen reduction test per the National Institutes of Health consensus definition (5), and late sepsis as a positive blood culture after $72 \mathrm{~h}$ of life.

\section{Biomarkers of Inflammation (Cytokines, LTA, and Endotoxin (LPS)) Level Determination}

TA specimens were centrifuged, and levels of interleukins (IL-1 $\beta$, IL-6, IL-8, IL-10, and IL-12), tumor necrosis factor- $\alpha$, and interferon- $\gamma$ were measured using a multiplex high-sensitivity cytokine array (Milliplex MAP ELISA Kit) purchased from Millipore (Billerica, MA) performed according to manufacturer's instructions.

Quantitative determination of LTA concentration in TAs was performed by ELISA kit, from EIAab Science Company (Wuhan, China). LPS was measured by Limulus amebocyte lysate using the QCL-1000 endpoint chromogenic Limulus amebocyte lysate assay by Lonza (Walkersville, MD). All concentrations were normalized to total immunoglobulin A content. Immunoglobulin A was measured using the BN ProSpec nephelometric method from Siemens Healthcare (Erlangen, Germany). The intra-assay coefficient of variation of these assays was $<10 \%$.

\section{Statistical Analysis}

Specimens were classified according to various clinical variables related to the subject (BPD or not; chorioamnionitis exposure or not). Statistical analysis was performed using SPSS v.21 software by IBM (Armonk, NY). Biomarker level data sets were log transformed and subjected to "independent samples $t$-test." Spearman's rank correlation coefficient was performed for all biomarker levels and all genera at each time point. Time series (repeat measures ANOVA) were performed in cases where $n \geq 3$ for each group; some time points were excluded due to patient drop off between time points. However, most patients were assessed at more than one time point.

\section{ACKNOWLEDGMENTS}

The authors thank Paula Revell, the Texas Children's Microbiome Center, and the Microbiology Laboratory, especially Jessica K. Runge and Megan Amerson for their invaluable help with specimen processing, and Michelle RubioGonzalez for database management. This work would not have been made possible without the assistance of bedside nurses and respiratory therapists delivering excellent patient care and aiding with specimen collection.

\section{STATEMENT OF FINANCIAL SUPPORT}

No financial assistance was received in support of the study.

Disclosure: The authors have no disclosures to make.

\section{REFERENCES}

1. Fanaroff AA, Stoll BJ, Wright LL, et al.; NICHD Neonatal Research Network. Trends in neonatal morbidity and mortality for very low birthweight infants. Am J Obstet Gynecol 2007;196:147.e1-8.

2. Stoll BJ, Hansen NI, Bell EF, et al.; Eunice Kennedy Shriver National Institute of Child Health and Human Development Neonatal Research Network. Neonatal outcomes of extremely preterm infants from the NICHD Neonatal Research Network. Pediatrics 2010;126:443-56.

3. Thome UH, Ambalavanan N. Permissive hypercapnia to decrease lung injury in ventilated preterm neonates. Semin Fetal Neonatal Med 2009;14:21-7.

4. Jobe AH, Bancalari E. Bronchopulmonary dysplasia. Am J Respir Crit Care Med 2001;163:1723-9.

5. Ehrenkranz RA, Walsh MC, Vohr BR, et al.; National Institutes of Child Health and Human Development Neonatal Research Network. Validation of the National Institutes of Health consensus definition of bronchopulmonary dysplasia. Pediatrics 2005;116:1353-60.

6. Bancalari E. Changes in the pathogenesis and prevention of chronic lung disease of prematurity. Am J Perinatol 2001;18:1-9.

7. Coalson JJ. Pathology of new bronchopulmonary dysplasia. Semin Neonatol 2003;8:73-81.

8. Kramer BW, Kallapur S, Newnham J, Jobe AH. Prenatal inflammation and lung development. Semin Fetal Neonatal Med 2009;14:2-7.

9. Viscardi RM, Muhumuza CK, Rodriguez A, et al. Inflammatory markers in intrauterine and fetal blood and cerebrospinal fluid compartments are associated with adverse pulmonary and neurologic outcomes in preterm infants. Pediatr Res 2004;55:1009-17.

10. D’Angio CT, Basavegowda K, Avissar NE, Finkelstein JN, Sinkin RA. Comparison of tracheal aspirate and bronchoalveolar lavage specimens from premature infants. Biol Neonate 2002;82:145-9.

11. Ramsay PL, DeMayo FJ, Hegemier SE, Wearden ME, Smith CV, Welty SE. Clara cell secretory protein oxidation and expression in premature infants who develop bronchopulmonary dysplasia. Am J Respir Crit Care Med 2001;164:155-61.

12. Watterberg KL, Demers LM, Scott SM, Murphy S. Chorioamnionitis and early lung inflammation in infants in whom bronchopulmonary dysplasia develops. Pediatrics 1996;97:210-5.

13. Jobe AH, Kallapur SG. Chorioamnionitis, surfactant, and lung disease in very low birth weight infants. J Pediatr 2010;156:3-4.

14. Van Marter LJ, Dammann O, Allred EN, et al.; Developmental Epidemiology Network Investigators. Chorioamnionitis, mechanical ventilation, and postnatal sepsis as modulators of chronic lung disease in preterm infants. J Pediatr 2002;140:171-6.

15. Beeton ML, Maxwell NC, Davies PL, et al. Role of pulmonary infection in the development of chronic lung disease of prematurity. Eur Respir J 2011;37:1424-30.

16. Cordero L, Ayers LW, Davis K. Neonatal airway colonization with gramnegative bacilli: association with severity of bronchopulmonary dysplasia. Pediatr Infect Dis J 1997;16:18-23.

17. Schelonka RL, Waites KB. Ureaplasma infection and neonatal lung disease. Semin Perinatol 2007;31:2-9.

18. Couroucli XI, Welty SE, Ramsay PL, et al. Detection of microorganisms in the tracheal aspirates of preterm infants by polymerase chain reaction: association of adenovirus infection with bronchopulmonary dysplasia. Pediatr Res 2000;47:225-32.

19. Ballard HO, Shook LA, Bernard P, et al. Use of azithromycin for the prevention of bronchopulmonary dysplasia in preterm infants: a randomized, double-blind, placebo controlled trial. Pediatr Pulmonol 2011;46:111-8.

20. Rogers GB, Carroll MP, Bruce KD. Studying bacterial infections through culture-independent approaches. J Med Microbiol 2009;58(Pt 11): 1401-18.

21. Rogers GB, Daniels TW, Tuck A, et al. Studying bacteria in respiratory specimens by using conventional and molecular microbiological approaches. BMC Pulm Med 2009;9:14.

22. Petrosino JF, Highlander S, Luna RA, Gibbs RA, Versalovic J. Metagenomic pyrosequencing and microbial identification. Clin Chem 2009;55:856-66.

23. Hamady M, Knight R. Microbial community profiling for human microbiome projects: tools, techniques, and challenges. Genome Res 2009;19:1141-52.

24. Hattori M, Taylor TD. The human intestinal microbiome: a new frontier of human biology. DNA Res 2009;16:1-12.

25. Nasution TA, Cheong SF, Lim CT, Leong EW, Ngeow YF. Multiplex PCR for the detection of urogenital pathogens in mothers and newborns. Malays J Pathol 2007;29:19-24.

26. Stressmann FA, Connett GJ, Goss K, et al. The use of culture-independent tools to characterize bacteria in endo-tracheal aspirates from pre-term infants at risk of bronchopulmonary dysplasia. J Perinat Med 2010;38:3337.

27. Mourani PM, Harris JK, Sontag MK, Robertson CE, Abman SH. Molecular identification of bacteria in tracheal aspirate fluid from mechanically ventilated preterm infants. PLoS One 2011;6:e25959. 
28. DiGiulio DB, Romero R, Amogan HP, et al. Microbial prevalence, diversity and abundance in amniotic fluid during preterm labor: a molecular and culture-based investigation. PLoS One 2008;3:e3056.

29. Nathe KE, Parad R, Van Marter LJ, et al. Endotoxin-directed innate immunity in tracheal aspirates of mechanically ventilated human neonates. Pediatr Res 2009;66:191-6.

30. Palmer C, Bik EM, DiGiulio DB, Relman DA, Brown PO. Development of the human infant intestinal microbiota. PLoS Biol 2007;5:e177.

31. Mshvildadze M, Neu J, Mai V. Intestinal microbiota development in the premature neonate: establishment of a lasting commensal relationship? Nutr Rev 2008;66:658-63.

32. Sharma R, Young C, Neu J. Molecular modulation of intestinal epithelial barrier: contribution of microbiota. J Biomed Biotechnol 2010;2010 305879.

33. Aderem A, Ulevitch RJ. Toll-like receptors in the induction of the innate immune response. Nature 2000;406:782-7.
34. Cho I, Blaser MJ. The human microbiome: at the interface of health and disease. Nat Rev Genet 2012;13:260-70.

35. Hilty M, Burke C, Pedro H, et al. Disordered microbial communities in asthmatic airways. PLoS One 2010;5:e8578.

36. Jumpstart Consortium Human Microbiome Project Data Generation Working Group. Evaluation of $16 \mathrm{~S}$ rDNA-based community profiling for human microbiome research. PLoS One 2012;7:e39315.

37. Caporaso JG, Kuczynski J, Stombaugh J, et al. QIIME allows analysis of high-throughput community sequencing data. Nat Methods 2010;7:335-6.

38. Riehle K, Coarfa C, Jackson A, et al. The Genboree Microbiome Toolset and the analysis of $16 \mathrm{~S}$ rRNA microbial sequences. BMC Bioinformatics 2012;13:Suppl 13:S11.

39. Li W, Godzik A. Cd-hit: a fast program for clustering and comparing large sets of protein or nucleotide sequences. Bioinformatics 2006;22:1658-9.

40. Shannon CE, Weaver W. The Mathematical Theory of Communication. Urbana, IL: University of Illinois Press, 1949. 